\title{
On the widths of Stokes lines in Raman scattering from molecules adsorbed at metal surfaces and in molecular conduction junctions
}

\author{
Yi Gad* and Michael Galperint \\ Department of Chemistry $\&$ Biochemistry, \\ University of California San Diego, La Jolla CA 92093, USA \\ Abraham Nitzan $\ddagger$ \\ Department of Chemistry, University of Pennsylvania, Philadelphia, PA 19104, USA and \\ School of Chemistry, Tel Aviv University, Tel Aviv, 69978, Israel
}

\begin{abstract}
Within a generic model we analyze the Stokes linewidth in surface enhanced Raman scattering (SERS) from molecules embedded as bridges in molecular junctions. We identify four main contributions to the off-resonant Stokes signal and show that under zero voltage bias (a situation pertaining also to standard SERS experiments) and at low bias junctions only one of these contributions is pronounced. The linewidth of this component is determined by the molecular vibrational relaxation rate, which is dominated by interactions with the essentially bosonic thermal environment when the relevant molecular electronic energy is far from the metal(s) Fermi energy(ies). It increases when the molecular electronic level is close to the metal Fermi level so that an additional vibrational relaxation channel due to electron-hole (eh) excition in the molecule opens. Other contributions to the Raman signal, of considerably broader linewidths, can become important at larger junction bias.
\end{abstract}

\footnotetext{
* yig057@ucsd.edu

$\dagger$ migalperin@ucsd.edu

$\ddagger$ nitzan@post.tau.ac.il
} 


\section{INTRODUCTION}

Molecular optoelectronics is an active field of research made possible by advances in laser technology and nanofabrication. [1] The possibility to conduct optical measurements in open non-equilibrium nano-systems resulted in the appearance of new diagnostic tools, and offers a route to optical control schemes such as switching in molecular electronics devices. Standard observables of optical spectroscopy can yield new information when monitored in open current-carrying molecular junctions. For example, current-induced fluorescence[2, 3] yields information on molecular resonances in the non-equilibrium system and makes imaging at submolecular resolution feasible, while the intensity of the emitted light corresponds to charge current noise at optical frequencies [4, 5] and can yield information on fast voltage transients at the tunnel junction. [6] Raman spectroscopy of current-carrying junctions can serve as a diagnostic tool similar to inelastic tunneling spectroscopy, and as an indicator for current-induced heating of electronic and vibrational degrees of freedom. [7-9] (Possible pitfalls of such characterization were discussed theoretically [10, 11]). Recently, measurements of $d c$ current and/or noise in response to laser pulse pair sequence was suggested as a variant of pump-probe spectroscopy for molecular junctions capable of providing information on intra-molecular dynamics at sup-picosecond timescale. [12, 13]

As noted above, the ability to characterize vibrational structure of a molecular device makes Raman scattering similar to inelastic electron tunneling spectroscopy (IETS). The corresponding spectra are characterized by their peak positions and heights, as well as lineshapes and linewidths. In addition to standard peaks, rich IETS lineshape features caused by interference between elastic and inelastic scattering channels are known. [14, 15] Similar interference features in Raman scattering were recently discussed.[16] The dependence of (resonant) IETS spectra on gate and source-drain biases was measured and discussed. [17-19] It appears to primarily manifest the sensitivity of molecular normal modes to the molecule charging state. [20, 21] Similarly, a shift in the frequencies of Stokes lines with bias was observed [9, 22] and was shown to result at least partly from the voltage dependence of the charge on the molecule. [23 26] Finally, the linewidths of IETS signals where studied both experimentally[27] and theoretically[28] and were shown to be dominated by the strength of electron-phonon interactions. No such study has been done so far for Raman scattering from molecular junctions. 
The present paper focuses on the latter issue: we identify the main contribution to the observed Raman intensity and analyze, using a generic model, the non-monotonic dependence of the Stokes linewidth on the gate and bias potentials. In Section [1] we introduce our model for an illuminated molecular junction as well as our calculation methodology for offresonant Raman scattering from this system. Section [II presents our results and Section IV concludes.

\section{MODEL AND METHOD}

We consider junction comprised of a molecule coupled to two metallic contacts, $L$ and $R$, each at its own equilibrium. The molecule is represented by two electronic levels (ground, $\varepsilon_{g}$, and excited, $\varepsilon_{x}$, states) and a molecular vibration, taken harmonic of frequency $\omega_{v}$, linearly coupled to the levels populations (an Holstein-type model). The junction is subjected to an

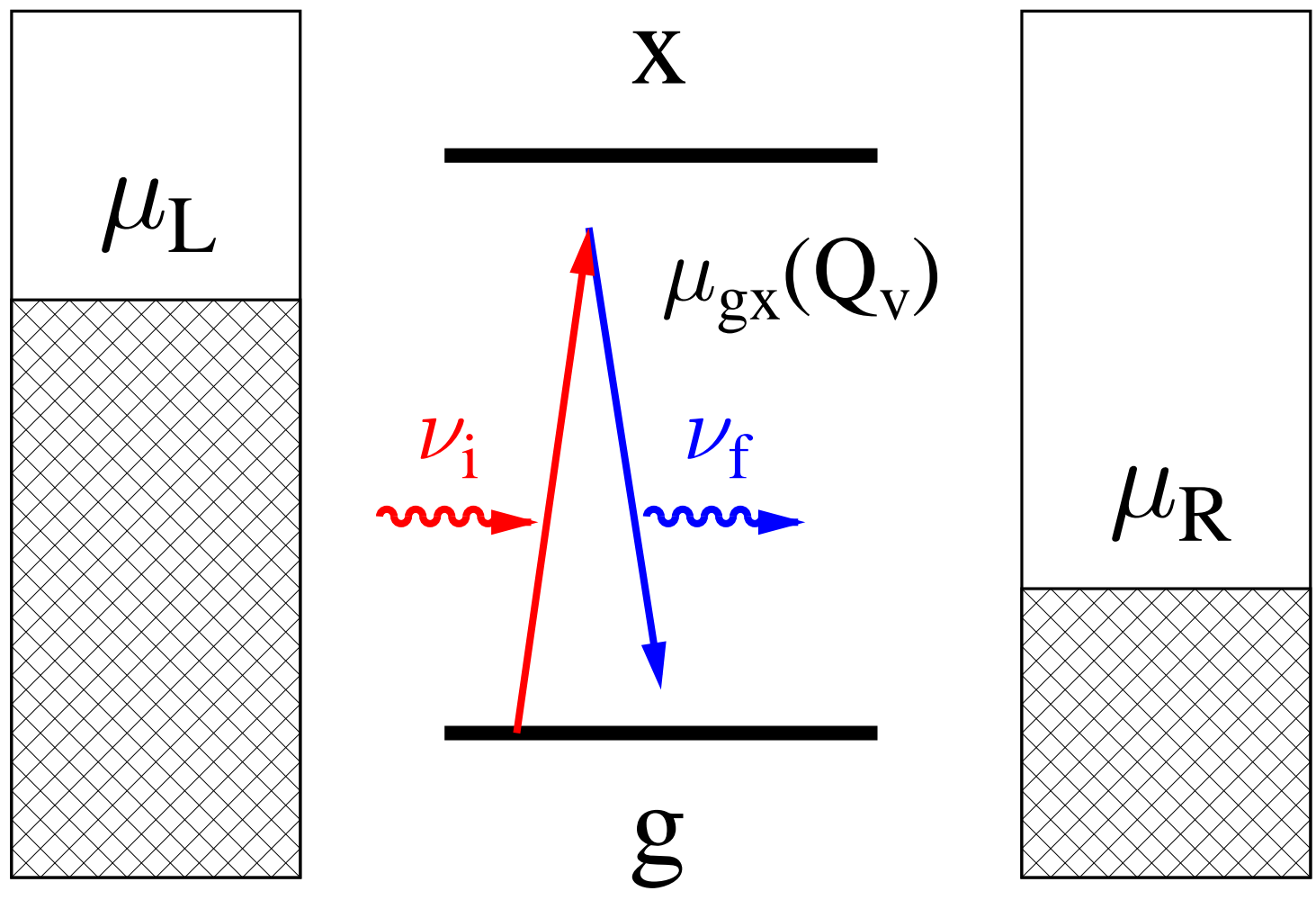

FIG. 1. (Color online) A sketch of the model for off-resonant Raman scattering in a junction. 
external radiation field, represented by a set of quantum harmonic modes $\left\{\nu_{\alpha}\right\}$ (see sketch in Fig. 11). One of these modes, of frequency $\nu_{i}$ represent the incident mode that pumps the system. All other modes, $\left\{\nu_{f}\right\}$, are taken to be vacant. The Hamiltonian is

$$
\hat{H}=\hat{H}_{0}+\hat{H}_{\text {rad }}+\hat{V}
$$

where $\hat{H}_{0}$ represents the dark junction, $\hat{H}_{\text {rad }}$ is Hamiltonian of the radiation field, and $\hat{V}$ is the molecule-field coupling. Explicitly

$$
\begin{aligned}
& \hat{H}_{0}= \sum_{m=g, x} \varepsilon_{m} \hat{n}_{m}+\omega_{v} \hat{v}^{\dagger} \hat{v}+\sum_{k} \varepsilon_{k} \hat{n}_{k}+\sum_{\beta} \omega_{\beta} \hat{b}_{\beta}^{\dagger} \hat{b}_{\beta} \\
&+\sum_{k, m}\left(V_{k m} \hat{c}_{k}^{\dagger} \hat{d}_{m}+H . c .\right)+\sum_{m=g, x} M_{m} \hat{Q}_{v} \hat{n}_{m} \\
&+\sum_{b e t a} V_{\beta}^{t h} \hat{Q}_{\beta} \hat{Q}_{v} \\
& \hat{H}_{\text {rad }}= \sum_{\alpha \in i\{f\}} \nu_{\alpha} \hat{a}_{\alpha}^{\dagger} \hat{a}_{\alpha} \\
& \hat{V}=\sum_{\alpha}\left(U_{\alpha D}\left(\hat{Q}_{v}\right) \hat{a}_{\alpha}^{\dagger} \hat{D}+H . c .\right)
\end{aligned}
$$

Here $\hat{d}_{m}^{\dagger}\left(\hat{d}_{m}\right)$ and $\hat{c}_{k}^{\dagger}\left(\hat{c}_{k}\right)$ create (annihilate) electrons in the molecular level $m$ and state $k$ of the metal contacts, respectively. $\hat{n}_{m}=\hat{d}_{m}^{\dagger} \hat{d}_{m}$ and $\hat{n}_{k}=\hat{c}_{k}^{\dagger} \hat{c}_{k}$ are the corresponding electron number operators for states $m(=g, x)$ of the molecule and $k$ of the contacts. $\hat{D}^{\dagger}=\hat{d}_{x}^{\dagger} \hat{d}_{g}$ and $\hat{D}=\hat{d}_{g}^{\dagger} \hat{d}_{x}$ are molecular excitation and de-excitation operators. $\hat{v}^{\dagger}(\hat{v})$ and $\hat{b}_{\beta}^{\dagger}\left(\hat{b}_{\beta}\right)$ create (annihilate) vibrational quanta in the molecule and mode $\beta$ of the thermal bath, respectively. $\hat{Q}_{v}=\hat{v}+\hat{v}^{\dagger}$ and $\hat{Q}_{v}=\hat{b}_{\beta}+\hat{b}_{\beta}^{\dagger}$ are the oscillators position operators. $\hat{a}_{\alpha}^{\dagger}\left(\hat{a}_{\alpha}\right)$ creates (destroys) photon in the mode $\alpha$ of radiation field. Note that this model contains two interactions that can cause inelastic light scattering. First is the dependence of the molecule-field coupling $U$ on the vibrational coordinate. The other is the polaronic coupling term in Eq.(2) whose importance is measured by the electron-vibration coupling M.

Following Refs. [29, 30] and focusing on the low voltage bias regime, we consider only 'normal Raman' scattering, i.e. a process where the initial state is its ground state. [31]

Raman scattering is a coherent process of fourth order in the matter-radiation field coupling (two orders correspond to the outgoing photon, blue line in Fig. 1, and two orders correspond to the incoming photon, red line in Fig. 1). Explicit steady-state expression for 
the 'normal Raman' scattering from the initial mode $i$ to a final mode $f$ of the radiation field is (see Ref. [30] for details)

$$
\begin{aligned}
& J_{i \rightarrow f}=\int_{-\infty}^{+\infty} d\left(t^{\prime}-t\right) \int_{-\infty}^{0} d\left(t_{1}-t\right) \int_{-\infty}^{0} d\left(t_{2}-t^{\prime}\right) \\
& e^{-i \nu_{f}\left(t^{\prime}-t\right)} e^{-i \nu_{i}\left(t_{1}-t_{2}\right)} \times \\
& \left\langle\hat{U}_{i D}\left(t_{2}\right) \hat{D}\left(t_{2}\right) \hat{U}_{D f}\left(t^{\prime}\right) \hat{D}^{\dagger}\left(t^{\prime}\right) \hat{U}_{f D}(t) \hat{D}(t) \hat{U}_{D i}\left(t_{1}\right) \hat{D}^{\dagger}\left(t_{1}\right)\right\rangle
\end{aligned}
$$

where $\hat{U}_{\alpha D} \equiv U_{\alpha D}\left(\hat{Q}_{v}\right)$. As in standard treatments, we expand the molecule-field coupling to linear term in Taylor series in the molecular vibrational displacement

$$
U_{\alpha D}\left(\hat{Q}_{v}\right) \approx U_{\alpha D}^{(0)}+U_{\alpha D}^{(1)} \hat{Q}_{v}
$$

Depending on combination of molecule-field coupling terms $\left(U_{\alpha D}^{(0)}\right.$ or $\left.U_{\alpha D}^{(1)} \hat{Q}_{v}\right)$ in the expression (5) one gets contributions to vibrational and electronic Raman (Rayleigh) scatterings. For example, substituting only $U_{\alpha D}^{(0)}$ in place of all molecule-field couplings in Eq.(5) yields the pure electronic Raman contribution discussed in Refs. [10, 11]. Here we focus on the vibrational Raman scattering, whose lowest order contribution comes from terms that are second order in the coupling to the molecular vibration. Such terms will be of order $\left(U^{(1)}\right)^{2}$. After collecting all such contributions to the vibrational Raman we (a) separate vibrational and electronic degrees of freedom (i.e. neglecting vibration-induced electronic correlations) and (b) neglect electronic correlation between ground and excited states of the molecule assuming that the energy gap between them is much larger than the widths associated with their coupling to the contacts. We focus on off-resonant Raman scattering and restrict our consideration to gate voltages that keep the upper electronic level above the leads chemical potentials (so it is essentially unpopulated), Under these approximations the explicit expression becomes

$$
\begin{aligned}
& J_{\nu_{i} \rightarrow \nu_{f}}=\rho\left(\nu_{i}\right) \Delta \nu_{i} \rho\left(\nu_{f}\right) \Delta \nu_{f} \operatorname{Re} \int \frac{d E_{g 1}}{2 \pi} \int \frac{d E_{g 2}}{2 \pi} \int \frac{d E_{x 1}}{2 \pi} \int \frac{d E_{x 2}}{2 \pi}\{ \\
& i D^{>}\left(\nu_{i f}\right) G_{g}^{<}\left(E_{g 1}\right) G_{g}^{<}\left(E_{g 2}\right) G_{x}^{>}\left(E_{x 1}\right) G_{x}^{>}\left(E_{x 2}\right) \frac{2 U_{i D}^{(0)} U_{D f}^{(1)} U_{f D}^{(0)} U_{D i}^{(1)}+U_{i D}^{(1)} U_{D f}^{(0)} U_{f D}^{(0)} U_{D i}^{(1)}+U_{i D}^{(0)} U_{D f}^{(1)} U_{f D}^{(1)} U_{D i}^{(0)}}{\left(\nu_{f}-E_{x 2}+E_{g 2}+i \delta\right)\left(\nu_{i}-E_{x 1}+E_{g 1}-i \delta\right)}
\end{aligned}
$$




$$
\begin{gathered}
-i D^{>}\left(\nu_{i f}-E_{g 21}\right) G_{g}^{<}\left(E_{g 1}\right) G_{g}^{>}\left(E_{g 2}\right) G_{x}^{>}\left(E_{x 1}\right) G_{x}^{>}\left(E_{x 2}\right)\left(\frac{2 U_{i D}^{(0)} U_{D f}^{(1)} U_{f D}^{(0)} U_{D i}^{(1)}}{\left(\nu_{f}-E_{x 2}+E_{g 2}+i \delta\right)\left(\nu_{i}-E_{x 1}+E_{g 1}-i \delta\right)}\right. \\
\left.+\frac{U_{i D}^{(1)} U_{D f}^{(0)} U_{f D}^{(0)} U_{D i}^{(1)}}{\left(\nu_{f}-E_{x 2}+E_{g 2}+i \delta\right)\left(\nu_{f}-E_{x 1}+E_{g 2}-i \delta\right)}+\frac{U_{i D}^{(0)} U_{D f}^{(1)} U_{f D}^{(1)} U_{D i}^{(0)}}{\left(\nu_{i}-E_{x 2}+E_{g 1}+i \delta\right)\left(\nu_{i}-E_{x 1}+E_{g 1}-i \delta\right)}\right) \\
-i D^{>}\left(\nu_{i f}-E_{x 21}\right) G_{g}^{<}\left(E_{g 1}\right) G_{g}^{<}\left(E_{g 2}\right) G_{x}^{<}\left(E_{x 1}\right) G_{x}^{>}\left(E_{x 2}\right)\left(\frac{2 U_{i D}^{(0)} U_{D f}^{(1)} U_{f D}^{(0)} U_{D i}^{(1)}}{\left(\nu_{f}-E_{x 1}+E_{g 2}+i \delta\right)\left(\nu_{i}-E_{x 2}+E_{g 1}-i \delta\right)}\right. \\
\left.+\frac{U_{i D}^{(0)} U_{D f}^{(1)} U_{f D}^{(1)} U_{D i}^{(0)}}{\left(\nu_{f}-E_{x 1}+E_{g 2}+i \delta\right)\left(\nu_{f}-E_{x 1}+E_{g 1}-i \delta\right)}+\frac{2\left(\nu^{2}\right)}{\left(\nu_{i}-E_{x 2}+E_{g 2}+i \delta\right)\left(\nu_{i}-E_{x 2}+E_{g 1}-i \delta\right)}\right) \\
-i D^{>}\left(\nu_{i f}-E_{x 21}-E_{g 21}\right) G_{g}^{<}\left(E_{g 1}\right) G_{g}^{>}\left(E_{g 2}\right) G_{x}^{<}\left(E_{x 1}\right) G_{x}^{>}\left(E_{x 2}\right)\left(\frac{2 U_{i D}^{(0)} U_{D f}^{(1)} U_{f D}^{(0)} U_{D i}^{(1)}}{\left(\nu_{f}-E_{x 1}+E_{g 2}+i \delta\right)\left(\nu_{i}-E_{x 2}+E_{g 1}-i \delta\right)}\right. \\
\left.\left.+\frac{U_{i D}^{(1)} U_{D f}^{(0)} U_{f D}^{(0)} U_{D i}^{(1)}}{\left|\nu_{f}-E_{x 1}+E_{g 2}+i \delta\right|^{2}}+\frac{U_{i D}^{(0)} U_{D f}^{(1)} U_{f D}^{(1)} U_{D i}^{(0)}}{\left.\mid \nu_{i}-E_{x 2}+E_{g 1}+i \delta\right)\left.\right|^{2}}\right)\right\}
\end{gathered}
$$

Here $\nu_{i f}=\nu_{i}-\nu_{f}, E_{m 21}=E_{m 2}-E_{m 1}(m=g, x), G_{m}^{>/</ r}(E)$ and $D^{>}(\omega)$ are Fourier transforms of the greater/lesser/retarded projections of the single electron Green function and the greater projection of the phonon Green function, respectively

$$
\begin{aligned}
G_{m}\left(\tau, \tau^{\prime}\right) & =-i\left\langle T_{c} \hat{d}_{m}(\tau) \hat{d}_{m}^{\dagger}\left(\tau^{\prime}\right)\right\rangle \\
D\left(\tau, \tau^{\prime}\right) & =-i\left\langle T_{c} \hat{Q}_{v}(\tau) \hat{Q}_{v}\left(\tau^{\prime}\right)\right\rangle
\end{aligned}
$$

where $T_{c}$ is the contour ordering operator. $\rho(\nu) \equiv \nu^{2} / \pi^{2} c^{3}$ is the density of optical modes.

Next, some simplification can be made by invoking the reasonable assumption that the molecule-contacts coupling is much larger than the molecule-radiation field coupling as well as the electron-phonon interaction. Under this assumption we can disregard the latter interactions in the expressions for the electronic Green functions, taking the forms that correspond to a molecule coupled to the two metal leads

$$
\begin{aligned}
& G_{m}^{r}(E)=\left[E-\varepsilon_{m}+i \Gamma_{m} / 2\right]^{-1} \\
& G_{m}^{<}(E)=i \frac{\Gamma_{m}^{L} f_{L}(E)+\Gamma_{m}^{R} f_{R}(E)}{\left(E-\varepsilon_{m}\right)^{2}+\left(\Gamma_{m} / 2\right)^{2}} \\
& G_{m}^{>}(E)=-i \frac{\Gamma_{m}^{L}\left[1-f_{L}(E)\right]+\Gamma_{m}^{R}\left[1-f_{R}(E)\right]}{\left(E-\varepsilon_{m}\right)^{2}+\left(\Gamma_{m} / 2\right)^{2}}
\end{aligned}
$$



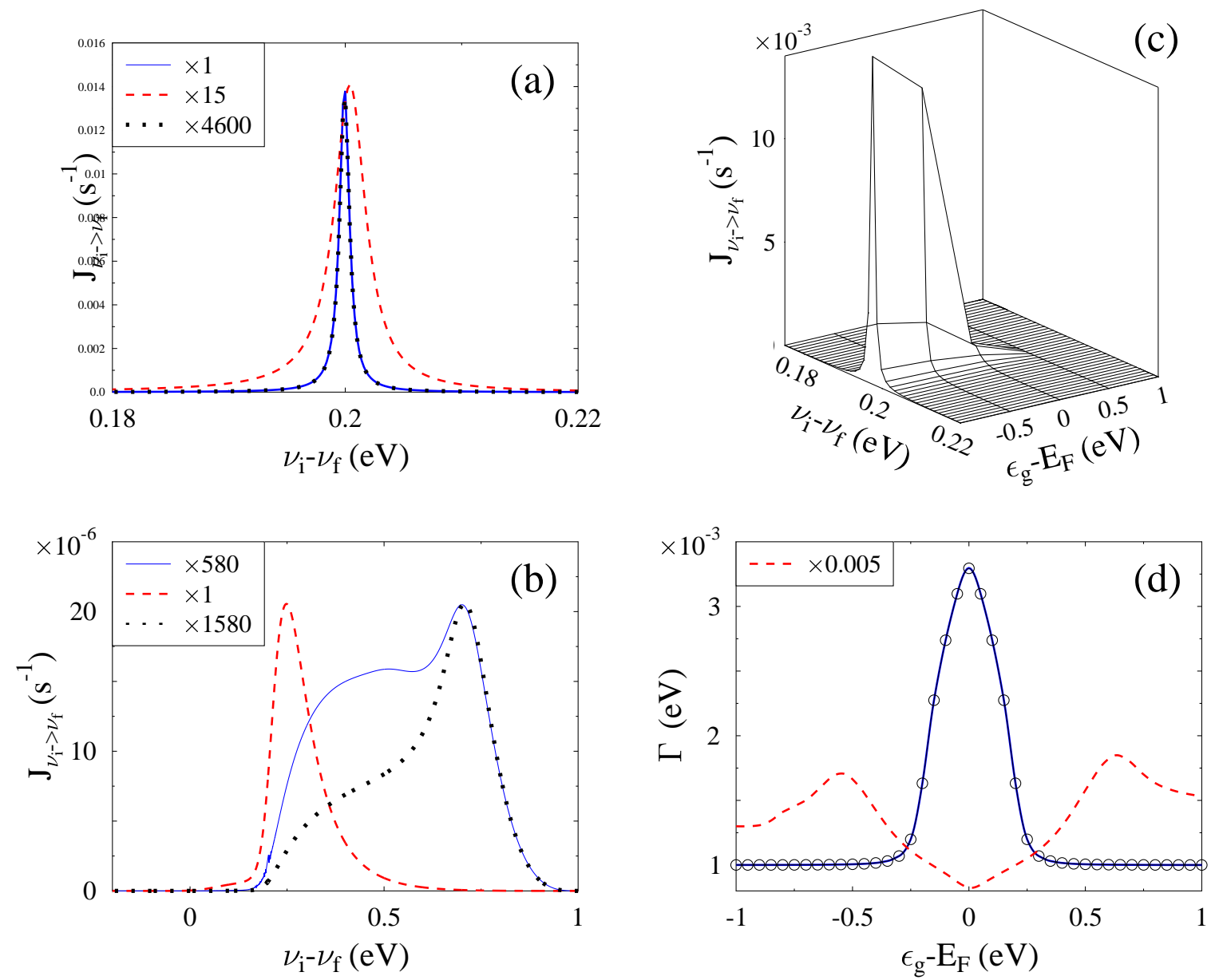

FIG. 2. (Color online) The Stokes component of the vibrational Raman scattering at equilibrium, $\mu_{L}=\mu_{R}=E_{F}$, for $\Gamma_{m}^{K}=0.05 \mathrm{eV}$. Shown are the contributions of (a) Eq. (7a) and (b) Eq. (7b) vs. Raman shift for three level positions: $\left(\varepsilon_{g}-E_{F}=-0.5 \mathrm{eV}\right.$, solid line, blue), at $\left(\varepsilon_{g}-E_{F}=0\right.$, dashed line, red) and above $\left(\varepsilon_{g}-E_{F}=0.5 \mathrm{eV}\right.$, dotted line, black). The total Stokes signal, Eq. (5), as function of the Raman shift and level position is shown in panel (c). Panel (d) shows widths $\Delta \nu$ (standard deviations) of the two main contributions (Eq. (7a $)$ - solid line, blue; Eq. (7b) - dashed line, red) as functions of the level position. Circles indicate broadening of the molecular vibration due to coupling to electron-hole excitations. See text for other parameters.

Here $\Gamma_{m}^{K} \equiv 2 \pi \sum_{k \in K}\left|V_{m k}\right|^{2} \delta\left(E-\varepsilon_{k}\right)(m=g, x, K=L, R)$ is electron escape rate from level $m$ into contact $K, \Gamma_{m}=\Gamma_{m}^{L}+\Gamma_{m}^{R}, f_{K}(E)$ is the Fermi-Dirac thermal distribution in contact $K=L, R$.

For the evaluation of the phonon Green functions we again disregard the molecule- 

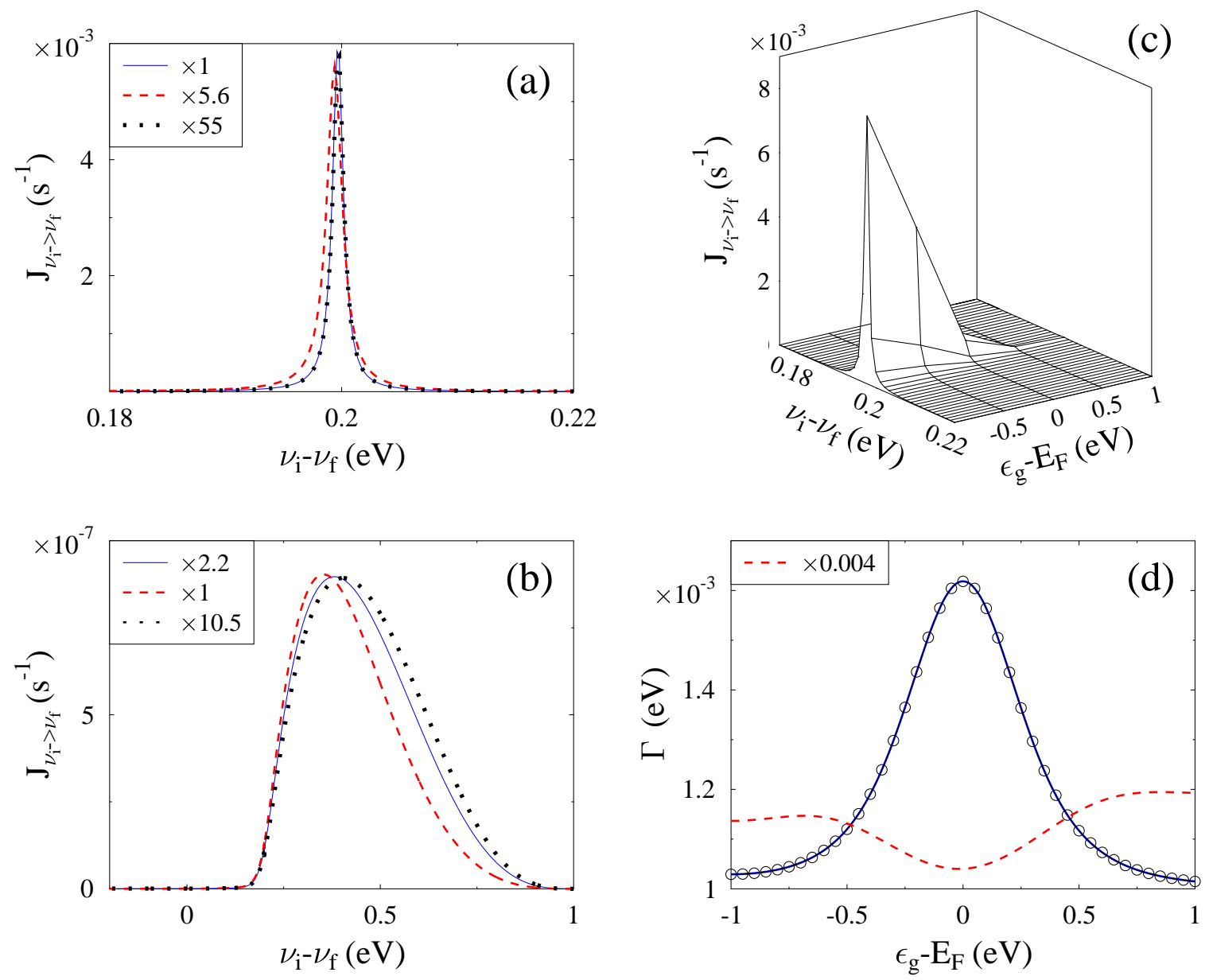

FIG. 3. (Color online) The Stokes component of the vibrational Raman scattering at equilibrium, $\mu_{L}=\mu_{R}=E_{F}$, for $\Gamma_{m}^{K}=0.4 \mathrm{eV}$. Shown are the contributions of (a) Eq. (7a) and (b) Eq. (7b) vs. Raman shift for three level positions: $\left(\varepsilon_{g}-E_{F}=-0.5 \mathrm{eV}\right.$, solid line, blue), at $\left(\varepsilon_{g}-E_{F}=0\right.$, dashed line, red) and above $\left(\varepsilon_{g}-E_{F}=0.5 \mathrm{eV}\right.$, dotted line, black). The total Stokes signal, Eq. (5)), as function of the Raman shift and level position is shown in panel (c). Panel (d) shows widths $\Delta \nu$ (standard deviations) of the two main contributions (Eq. (7a $)$ - solid line, blue; Eq. (7b) - dashed line, red) as functions of the level position. Circles indicate broadening of the molecular vibration due to coupling to electron-hole excitations. See text for other parameters.

radiation field coupling, but keep the electron-phonon interaction. This leads to

$$
\begin{aligned}
D^{r}(\omega) & =\left[\left[D_{0}^{r}(\omega)\right]^{-1}-\Pi_{t h}^{r}(\omega)-\Pi_{e l}^{r}(\omega)\right]^{-1} \\
D^{>/<}(\omega) & =D^{r}(\omega)\left(\Pi_{t h}^{>/<}(\omega)+\Pi_{e l}^{>/<}(\omega)\right) D^{a}(\omega)
\end{aligned}
$$


We will henceforth assume that $\omega>0$ and use $D^{>/<}(-\omega)=D^{</>}(\omega)$ to access the $\omega<0$ region. In Eqs. (13) and (14) $D^{a}(\omega)=\left[D^{r}(\omega)\right]^{*}$,

$$
D_{0}^{r}(\omega)=\frac{1}{\omega-\omega_{v}+i \delta}-\frac{1}{\omega+\omega_{v}+i \delta}
$$

is the retarded projection of free phonon Green function, and

$$
\begin{aligned}
& \Pi_{t h}^{r}(\omega)=-i \frac{\gamma(\omega)}{2} \\
& \Pi_{t h}^{<}(\omega)=-i \gamma(\omega) N(\omega) \\
& \Pi_{t h}^{>}(\omega)=-i \gamma(\omega)[1+N(\omega)]
\end{aligned}
$$

are the projections of the self-energy of the molecular vibration due to its coupling to the (bosonic) white thermal bath. Here $N(\omega)$ is the Bose-Einstein thermal distribution and $\gamma(\omega)=2 \pi \sum_{\beta}\left|V_{\beta}^{t h}\right|^{2} \delta\left(\omega-\omega_{\beta}\right)$ is the dissipation rate of molecular vibrational excitation due to coupling to thermal bath. The self energy of the molecular phonon associated with the electron-vibration coupling is treated at the level of the Born approximation

$$
\begin{array}{r}
\Pi_{e l}^{r}(\omega)=-i M_{g}^{2} \int \frac{d E}{2 \pi}\left(G_{g}^{<}(E) G_{g}^{a}(E-\omega)\right. \\
\left.+G_{g}^{r}(E) G_{g}^{<}(E-\omega)\right) \\
\Pi_{e l}^{<}(\omega)=-i M_{g}^{2} \int \frac{d E}{2 \pi} G_{g}^{<}(E) G_{g}^{>}(E-\omega) \\
\Pi_{e l}^{>}(\omega)=-i M_{g}^{2} \int \frac{d E}{2 \pi} G_{g}^{>}(E) G_{g}^{<}(E-\omega)
\end{array}
$$

Before describing our numerical results, it is important to note the different physical origins of the four contributions, Eqs. (7ad)-(7d), to the Raman signal, that can be inferred from the different forms of the electronic Green functions appearing in them and the forms of the corresponding energy denominators. It is convenient to look at them in comparison to the pure electronic Raman components discussed in Ref. [11] (see Fig. 2 in this reference). Without the vibrational shift the contribution (7a) with $G_{g}^{<} G_{g}^{<} G_{x}^{>} G_{x}^{>}$would be the Rayleigh line where each scattering event involves a single electron-hole pair - an occupied electronic level near $E_{g}$ and an empty electronic level near $E_{x}$. The contribution (7b) with $G_{g}^{<} G_{g}^{>} G_{x}^{>} G_{x}^{>}$if considered without the vibrational shift corresponds to that contribution to the pure electronic Raman scattering where the difference between the initial and final photon energy is expressed by moving an electron between two metal levels close to $E_{g}$, requiring one of these 
levels to be occupied and the other empty. The term (17c) that depends on $G_{g}^{<} G_{g}^{<} G_{x}^{<} G_{x}^{>}$ is similar, except that the difference between incoming and outgoing photons is expressed in electron motion between two levels near $E_{x}$, again requiring one of them to be occupied and the other empty. Finally, the contribution (17d) that contains $G_{g}^{<} G_{g}^{>} G_{x}^{<} G_{x}^{>}$splits the photon energy difference between two electronic transitions, one near $E_{x}$ and the other near $E_{g}$.

Two observations follow, still on this qualitative level: First, in equilibrium and at low bias, in the common situation where the lower and upper electronic orbitals are far below and far above the metal(s) Fermi energy(ies) respectively, Eq. (17a) will be the dominant contribution to the vibrational Raman signal. Second, the vibrational Raman lines associated with this contribution will be narrow in the sense that their width will not reflect the excitation of electron-hole pairs in the metal. The contributions (7b) and (7c) will be important in situations where, respectively, $E_{g}$ and $E_{x}$ are close to the metals Fermi energies. Furthermore, these contributions will be considerable broader, reflecting the excitation of electron-hole pairs in the metal alongside the vibrational excitation. Note that at low temperatures this broadening will be asymmetric, corresponding to an electronic side-band of the vibrational Raman transition as recently discussed in Ref. [16]. Finally, we expect that also the pure vibrational Raman spectrum associated with Eq. (7a will be broader when one of the the molecular electronic levels is close to the Fermi energy, because of the increased importance of the electronic relaxation channel for the molecular vibration in this situation. [23]

\section{NUMERICAL RESULTS}

Here we present numerical results for the Raman flux, Eq. (17), for the model (11)-(4). Below we focus on the most prominent contributions, Eqs. (7ad) and (7b). The following parameters are used in these calculations: $T=100 \mathrm{~K}, \varepsilon_{x}-\varepsilon_{g}=2 \mathrm{eV}$ (the absolute level positions are varied as described below), $\Gamma_{m}^{L}=\Gamma_{m}^{R}=0.05 \mathrm{eV}$ (in Figs. 2 and 4) and $0.4 \mathrm{eV}$ (in Figs. 3] and 5), $(m=g, x), \omega_{v}=0.2 \mathrm{eV}, \gamma\left(\omega_{v}\right)=10^{-3} \mathrm{eV}$, and $M_{g}=0.03 \mathrm{eV}$. The Fermi energy is chosen as the origin, $E_{F}=0$, and the bias is applied symmetrically

$\mu_{L}=E_{F}+|e| V_{s d} / 2$ and $\mu_{R}=E_{F}-|e| V_{s d} / 2$. The incident frequency is taken as $\nu_{i}=1 \mathrm{eV}$, which corresponds for the present choice of molecular parameters to off-resonant Raman 
scattering. The couplings to the radiation field are assumed to satisfy $U_{\alpha D}^{(0)}=U_{\alpha D}^{(1)}=0.01 \mathrm{eV}$. The optical resolution windows of the incident energy and measuring device, $\Delta \nu_{i}$ and $\Delta \nu_{f}$ in Eq. (7), are taken to be the same, $0.01 \mathrm{eV}$. The calculations were performed on an energy grid spanning the range from -5 to $5 \mathrm{eV}$ with step size $5 \times 10^{-5} \mathrm{eV}$.

We envision an experiment in which the position of the molecular resonances can be changed by a gate voltage. We start from the situation where level $g$ is far below the Fermi energy and level $x$ is far above it, so that the lower level is occupied and upper one is empty, and consider the effect on the Raman spectrum of applying a gate voltage to move $\varepsilon_{g}$ to the vicinity of, and then beyond, the chemical potentials. In this regime the two main contributions to the Raman flux are given by Eqs. (7a) and (7b) with the first one dominating the intensity of the Stokes line. (As explained above, the terms (7c) and (7d) are potentially important only when the excited state is populated). The Raman linewidths reported below are estimated using the standard deviation associated with the corresponding Raman peak calculated on the employed energy grid.

Figure 2 shows results of of this calculation for the equilibrium case, $\mu_{L}=\mu_{R}=E_{F}$. Note that the intensity of the Stokes line decreases with decrease of the population in the ground state (see Fig. 2k), however the implication of this observation should be understood with respect to the 2-level model used here. In reality, when $\varepsilon_{g}$ goes up and above the metal Fermi energy, other lower molecular levels will contribute to the Raman signal. Disregarding this issue, the following additional observations can be made:

1. The dominant Raman feature is indeed that associated with contribution (7aa) the electronically elastic/vibrationally inelastic signal. The contribution (7b) becomes comparable when $\varepsilon_{g}$ is near the metal Fermi energy. It should be kept in mind that and additional broad feature, the electronically inelastic/vibrationally elastic (pure electronic) is not displayed in these figures. In experimental spectra, the signal (7b) may often become part of this broad electronic background.

2. The width of the contribution (7b $)$ is far greater than that of the electronically elastic term (7a), as long as $\varepsilon_{g}$ is far from the metal Fermi energy. However, the width of (7a) increases considerably when $\varepsilon_{g}$ approaches $E_{F}$.

3. The widths of the two contributions, (7a and (7b), behave symmetrically about the Fermi energy (see Figs. 2a and b). Such behavior is expected since in both cases the 
width is defined by convolution of electron and hole populations, $G_{g}^{<}\left(E_{g 1}\right) G_{g}^{>}\left(E_{g 2}\right)$, which at equilibrium is symmetric relative to the Fermi energy.

4. Comparing the results displayed in Figures 2 and 3 (small and large molecule-metal coupling $(\Gamma)$, respectively, we note that the dominant low bias feature, namely the contribution (7a) is essentially the same in both cases. Interestingly, when $\varepsilon_{g}$ is at the Fermi energy (dashed red lines in Figs. $2 \mathrm{a}$ and $3 \mathrm{a}$ ), this feature is broader in the smaller $\Gamma$ case. This is also seen in comparing Figs. $2 \mathrm{~d}$ and $3 \mathrm{~d}$. This behavior reflects the fact that when $\Gamma>k_{B} T$, even when $\varepsilon_{g}=E_{F}$, most of the electronic spectral density (of width $\Gamma)$ is outside the region of partial electronic occupation $(f(1-f) \neq 0$ where $\mathrm{f}$ is the Fermi distribution) in which the electronic channel for vibrational relaxation is open. The fact that the spectra in Fig. 33are smoother and less structured than in Fig. 2 similarly reflects the fact that for large $\Gamma$ all behaviors associated with the position of $\varepsilon_{g}$ relative to $E_{F}$ and the width of the partially populated region are smoothened.

The width of the vibrational Raman lines reflects three types of contributions. First there is the relaxation to the thermal bosonic environment that is not affected (in our model) by the bias and gate potentials. Second is the additional relaxation channel due to electronvibration coupling, that can dominate the overall width when the molecular electronic level approaches the metal Fermi level. The structure of this contribution suggests that the width of the term (7a) (solid line in Fig. 2 $2 \mathrm{~d}$ ) is dominated by the (renormalized) density of molecular vibration (circles in Fig. 2d d). Finally, as discussed above, there is the electron-hole sideband that appears prominently in the term (7b) (as well as (7c) and (7d) ). Note again that in actual observations it will not be easy to distinguish between this sideband to the vibrational transition and the underlying Raman continuum that originates primarily from the pure electronic Raman scattering. [11]

We now turn to the nonequilibrium situation with $\mu_{L}=0.5 \mathrm{eV}$ and $\mu_{R}=-0.5 \mathrm{eV}$. The total Stokes intensity is here affected by two factors: the population of the lower level and the current induced heating of the molecular vibration. As a result, the decrease in the Stokes intensity when $\varepsilon_{g}$ approaches the lowest chemical potential due to depletion of the level population changes to increase in the intensity when the level is in the bias window (nonequilibrium feature) - see Fig. 迎. The width of the dominant contribution (7a) shows similar behavior as in the equilibrium case, with increase of the width resulting from 
opening the electronic relaxation channel when $\varepsilon_{g}$ approaches the metal Fermi energies. This contribution to the width is again symmetric about each of the Fermi energies (see Figs. 4 and $3 \mathrm{~d}$ ). In contrast, the nonequilibrium electronic distribution, in particular the existence of two energy regions of partial populations of metal electronic states, causes drastic changes and more structure in both lineshape (Fig. 4b) and linewidth (Fig. 3d) of the contribution (7b) as compared to equilibrium case. This structure is again smoothened in the large $\Gamma$ case (Fig. [5). Still, since this peak is much lower and broader than that of (7a), it may be considered as part of the electronic Raman background.

\section{CONCLUSION}

Within a simple two-level model of a molecular junction we consider off-resonant Raman scattering and discuss dependence of Stokes linewidth on gate and bias voltages. We focus on low bias regime, where upper level is almost empty, and thus consider only "normal Raman' contribution to the total signal (i.e. Raman scattering which originates at the lower molecular level). Employing realistic parameters we show that the linewidth changes nonmonotonically with gate voltage demonstrating maximum at resonance between molecular

level and chemical potential(s) of metallic contacts. Analysis shows that the effect is due to opening of an electronic relaxation channel for molecular vibrations by which e-h excitations are formed in metallic contacts. At low biases and for realistic parameters this mechanism is the dominant contribution to the Stokes linewidth. Other mechanisms are relaxation of molecular vibration due to coupling to the thermal environment and surface plasmons. The latter was not included in the consideration due to mismatch between characteristic plasmons and molecular vibrations frequencies. Note that the model also disregards inhomogeneous broadening and pure dephasing contributions. Experimental verification of our theoretical prediction seems to be a realistic possibility.

\section{ACKNOWLEDGMENTS}

The research of AN is partially supported by the Israel Science Foundation and the US-Israel Binational Science Foundation. MG gratefully acknowledges support by the US 
Department of Energy.

[1] M. Galperin and A. Nitzan, Phys. Chem. Chem. Phys. 14, 9421 (2012).

[2] S. W. Wu, G. V. Nazin, and W. Ho, Phys. Rev. B 77, 205430 (2008).

[3] C. Chen, P. Chu, C. A. Bobisch, D. L. Mills, and W. Ho, Phys. Rev. Lett. 105, 217402 (2010).

[4] N. L. Schneider, G. Schull, and R. Berndt, Phys. Rev. Lett. 105, 026601 (2010).

[5] N. L. Schneider, J. T. Lü, M. Brandbyge, and R. Berndt, Phys. Rev. Lett. 109, 186601 (2012).

[6] C. Grosse, M. Etzkorn, K. Kuhnke, S. Loth, and K. Kern, Appl. Phys. Lett. 103, 183108 (2013).

[7] Z. Ioffe, T. Shamai, A. Ophir, G. Noy, I. Yutsis, K. Kfir, O. Cheshnovsky, and Y. Selzer, Nature Nanotech. 3, 727 (2008).

[8] D. R. Ward, N. J. Halas, J. W. Ciszek, J. M. Tour, Y. Wu, P. Nordlander, and D. Natelson, Nano Lett. 8, 919 (2008).

[9] D. R. Ward, D. A. Corley, J. M. Tour, and D. Natelson, Nature Nanotech. 6, 33 (2011).

[10] M. Galperin and A. Nitzan, J. Phys. Chem. Lett. 2, 2110 (2011).

[11] M. Galperin and A. Nitzan, Phys. Rev. B 84, 195325 (2011).

[12] Y. Selzer and U. Peskin, J. Phys. Chem. C 117, 22369 (2013).

[13] M. A. Ochoa, Y. Selzer, U. Peskin, and M. Galperin, J. Phys. Chem. Lett. 6, 470 (2015).

[14] M. Galperin, M. A. Ratner, and A. Nitzan, J. Chem. Phys. 121, 11965 (2004).

[15] D. Rai and M. Galperin, Phys. Rev. B 86, 045420 (2012).

[16] S. Dey, M. Banik, E. Hulkko, K. Rodriguez, V. A. Apkarian, M. Galperin, and A. Nitzan, Phys. Rev. B 93, 035411 (2016).

[17] H. Park, J. Park, A. K. L. Lim, E. H. Anderson, A. P. Alivisatos, and P. L. McEuen, Nature 407, 57 (2000).

[18] A. N. Pasupathy, J. Park, C. Chang, A. V. Soldatov, S. Lebedkin, R. C. Bialczak, J. E. Grose, L. A. K. Donev, J. P. Sethna, D. C. Ralph, and P. L. McEuen, Nano Lett. 5, 203 (2005).

[19] D.-H. Chae, J. F. Berry, S. Jung, F. A. Cotton, C. A. Murillo, and Z. Yao, Nano Lett. 6, 165 (2006).

[20] M. Galperin, A. Nitzan, and M. A. Ratner, Phys. Rev. B 78, 125320 (2008). 
[21] A. J. White and M. Galperin, Phys. Chem. Chem. Phys. 14, 13809 (2012).

[22] D. Natelson, Y. Li, and J. B. Herzog, Phys. Chem. Chem. Phys. 15, 5262 (2013).

[23] K. Kaasbjerg, T. Novotný, and A. Nitzan, Phys. Rev. B 88, 201405 (2013).

[24] Y. Li, P. Doak, L. Kronik, J. B. Neaton, and D. Natelson, Proc. Natl. Acad. Sci. 111, 1282 (2014).

[25] A. J. White, S. Tretiak, and M. Galperin, Nano Lett. 14, 699 (2014).

[26] A. J. White, M. A. Ochoa, and M. Galperin, J. Phys. Chem. C 118, 11159 (2014).

[27] W. Wang, T. Lee, I. Kretzschmar, and M. A. Reed, Nano Lett. 4, 643646 (2004).

[28] M. Galperin, M. A. Ratner, and A. Nitzan, Nano Lett. 4, 1605 (2004).

[29] M. Galperin, M. A. Ratner, and A. Nitzan, Nano Lett. 9, 758 (2009).

[30] M. Galperin, M. A. Ratner, and A. Nitzan, J. Chem. Phys. 130, 144109 (2009).

[31] For strongly biased junctions both lower and upper molecular states may be partially occupied, giving rise to more contributions to elastic and inelastic light scattering, see Ref. [30]. 

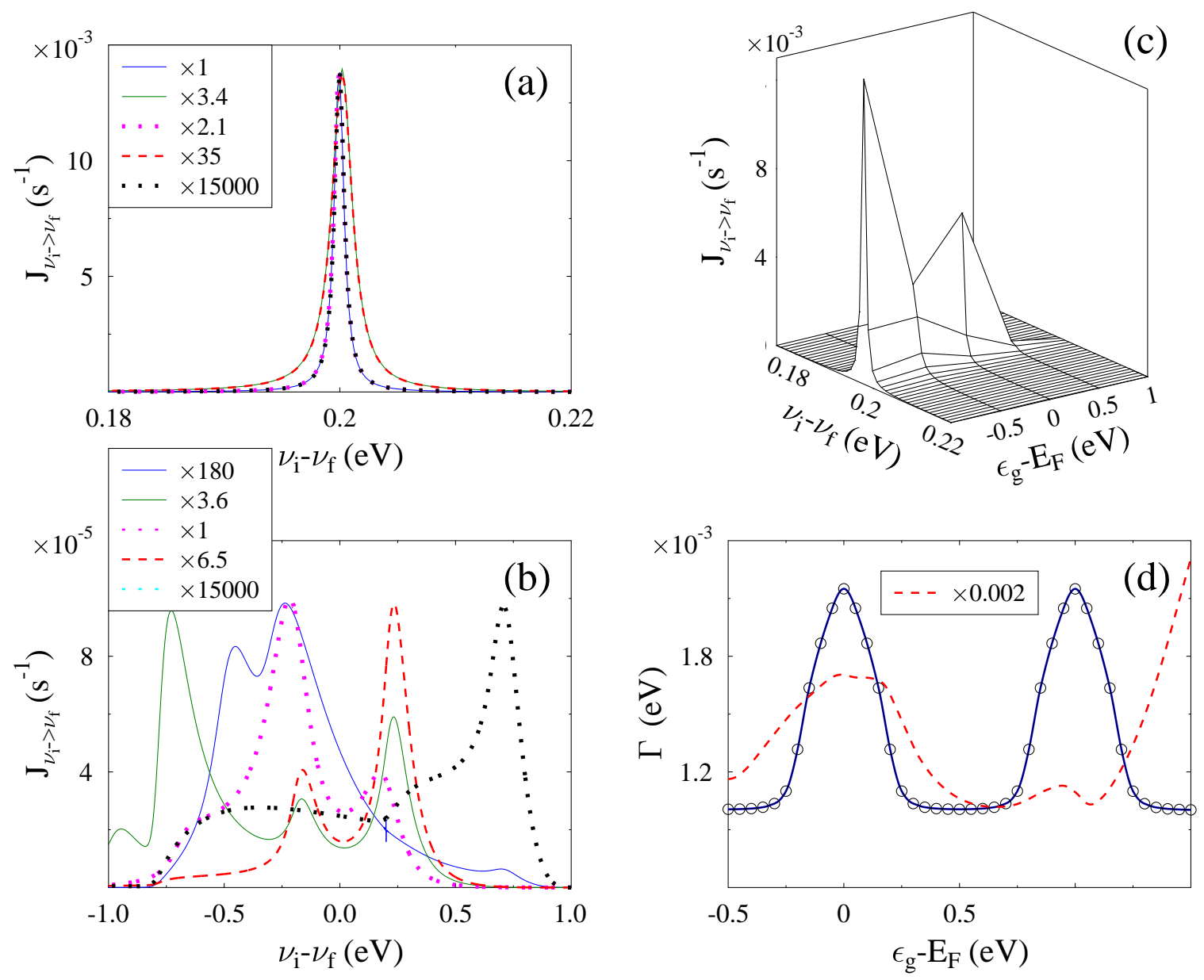

FIG. 4. (Color online) Stokes scattering from a biased junction, $\mu_{L}=0.5 \mathrm{eV}$ and $\mu_{R}=-0.5 \mathrm{eV}$ for $\Gamma_{m}^{K}=0.05 \mathrm{eV}$. Shown is the dependence of the Stokes signal on Raman shift for level position $\varepsilon_{g}<\mu_{R}\left(\varepsilon_{g}=-1 \mathrm{eV}\right.$, solid line, blue), $\varepsilon_{g}=\mu_{R}$ (solid line, green), $\mu_{R}<\varepsilon_{g}<\mu_{L}\left(\varepsilon_{g}=0\right.$, dotted line, magenta), $\varepsilon_{g}=\mu_{L}$ (dashed line, red), and $\varepsilon_{g}>\mu_{L}\left(\varepsilon_{g}=1 \mathrm{eV}\right.$, dotted line, black) for the contributions of (a) Eq. (7a) and (b) Eq. (7b). The Inset shows the scaling parameters used for the different lines. The total Stokes scattering, Eq. (5), as function of the Raman shift and level position is shown in panel (c). Panel (d) shows widths $\Delta \nu$ (standard deviations) of the two main contributions (Eq. (7a) - solid line, blue; Eq. (7b) - dashed line, red) as functions of the level position. Circles indicate broadening of the molecular vibration due to coupling to electron-hole excitations. See text for other parameters. 

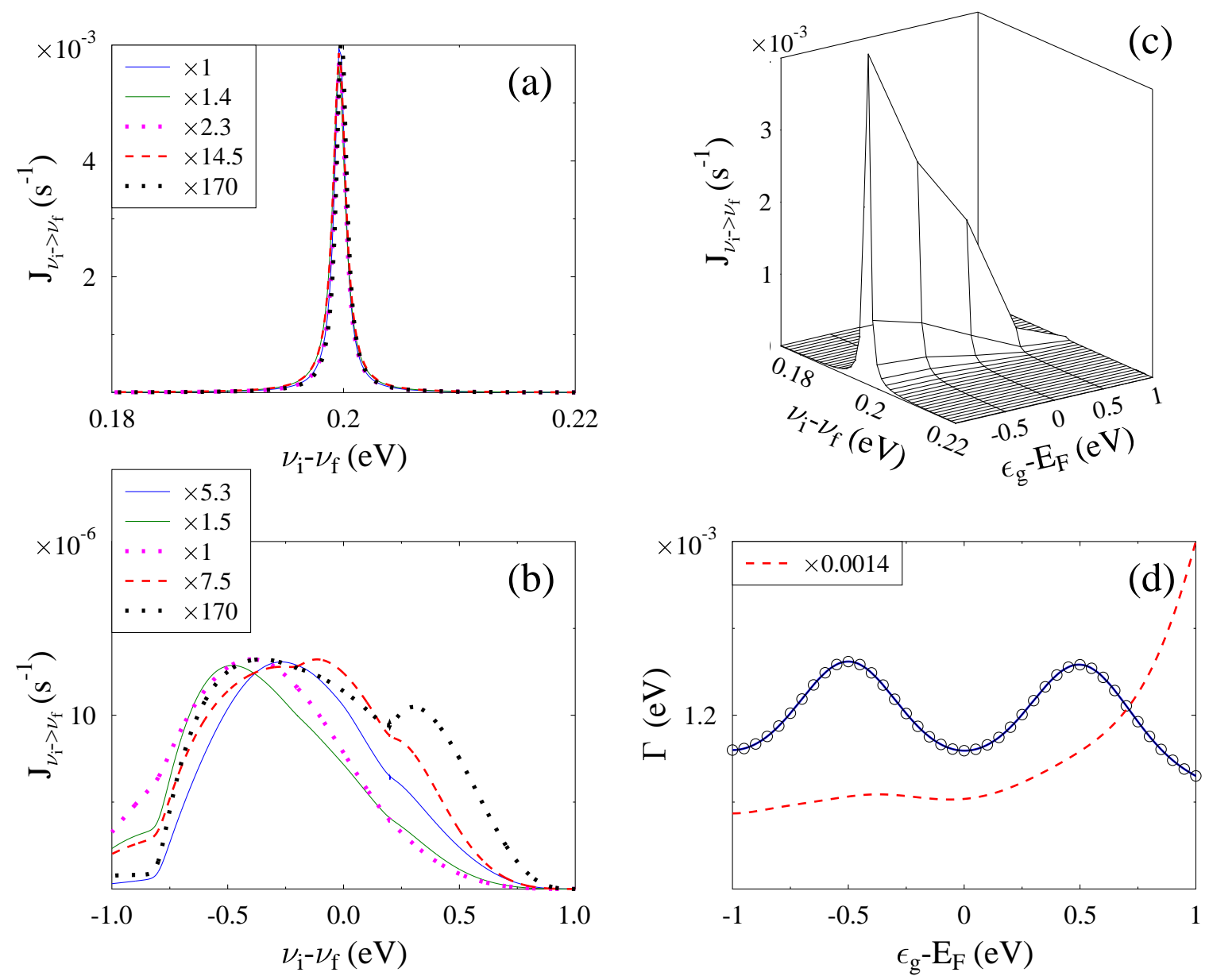

FIG. 5. (Color online) Stokes scattering from a biased junction, $\mu_{L}=0.5 \mathrm{eV}$ and $\mu_{R}=-0.5 \mathrm{eV}$, for $\Gamma_{m}^{K}=0.4 \mathrm{eV}$. Shown is the dependence of the Stokes signal on Raman shift for level position $\varepsilon_{g}<\mu_{R}\left(\varepsilon_{g}=-1 \mathrm{eV}\right.$, solid line, blue), $\varepsilon_{g}=\mu_{R}$ (solid line, green), $\mu_{R}<\varepsilon_{g}<\mu_{L}\left(\varepsilon_{g}=0\right.$, dotted line, magenta), $\varepsilon_{g}=\mu_{L}$ (dashed line, red), and $\varepsilon_{g}>\mu_{L}\left(\varepsilon_{g}=1 \mathrm{eV}\right.$, dotted line, black) for the contributions of (a) Eq. (7a) and (b) Eq. (7b). The Inset shows the scaling parameters used for the different lines. The total Stokes scattering, Eq. (5), as function of the Raman shift and level position is shown in panel (c). Panel (d) shows widths $\Delta \nu$ (standard deviations) of the two main contributions (Eq. (7a) - solid line, blue; Eq. (7b) - dashed line, red) as functions of the level position. Circles indicate broadening of the molecular vibration due to coupling to electron-hole excitations. See text for other parameters. 